\title{
SENSOR FUSION FOR 3D ARCHAEOLOGICAL DOCUMENTATION AND RECONSTRUCTION: CASE STUDY OF "CUEVA PINTADA" IN GALDAR, GRAN CANARIA
}

\author{
S. Del Pozo ${ }^{1, *}$, P. Rodríguez-Gonzálvez ${ }^{2}$, D. Hernández-López ${ }^{3}$, J. Onrubia-Pintado ${ }^{3}$, D. González-Aguilera ${ }^{1}$ \\ ${ }^{1}$ Dept. of Cartographic and Land Engineering, University of Salamanca, Hornos Caleros, 50, Ávila, Spain - (s.p.aguilera, \\ daguilera)@usal.es \\ ${ }^{2}$ Dept. of Mining Technology, Topography and Structures. Universidad de León, Avda. de Astorga, s/n, Ponferrada, León, Spain - \\ p.rodriguez@unileon.es \\ ${ }^{3}$ IDR, Institute for Regional Development, University of Castilla La-Mancha, Albacete, Spain - (david.hernandez, \\ jorge.onrubia)@uclm.es
}

Commission II, WG II/8

KEY WORDS: Photogrammetry, Laser Scanning, Virtual Tour, Archaeology, Structure from Motion, Computer Vision, Image-Based Modelling, Software Development

\begin{abstract}
:
3D documentation and reconstruction are decisive when inspecting archaeological sites without any direct contact or when monitoring the changes they have suffered over time are required. In addition, there is a great commitment with the preservation of heritage as well as with the awareness and dissemination of these historical sites. In this sense, this paper presents a visualization system with metric properties that will serve to manage and disseminate the archaeological site under study. Specifically, two techniques were used in an integrated way for the data acquisition: laser scanning and close-range photogrammetry. Both techniques come together in perfect marriage as remote data acquisition techniques to generate high quality $3 \mathrm{D}$ spatial products of this type of scenarios. A combined acquisition methodology is proposed in which the design of a network using reference spheres and targets is the key to link data from both technologies. This paper will describe the proposed methodology and the results obtained in the archaeological site of "Cueva Pintada" in Gran Canaria (Spain) which has an area of around $5400 \mathrm{~m}^{2}$. As final products: (i) a laser model of $\pm 1.68 \mathrm{~mm}$ accuracy and $5 \mathrm{~mm}$ resolution, (ii) a photorealistic photogrammetric model of $\pm 8.4 \mathrm{~mm}$ accuracy and $1.47 \mathrm{~mm}$ of spatial resolution thanks to a specific pole developed for this purpose and (iii) a virtual tour with metric properties of the whole archaeological site were obtained.
\end{abstract}

\section{INTRODUCTION}

\subsection{D Digitalization of Cultural Heritage}

Three-dimensional documentation and reconstruction of Cultural Heritage $(\mathrm{CH})$ is the key to digitally protect and preserve these historical sites (Patias et al., 2006; Megahed, 2015; Khodeir et al., 2016; Hua et al., 2018). In this sense, high resolution 3D models have been created to study a great variability of heritage environments: landscapes (Rubinowicz et al., 2015; Giannini et al., 2017), archaeological remains (Niccolucci et al., 2016; Themistocleous, 2017), caves (Lerma et al., 2010; Hoffmeister, 2017; Landeschi et al., 2019) and desert sites (Pozzi-Escot et al., 2018; Lucci et al., 2019) among others. Geotechnologies such as laser scanning, photogrammetry, multispectral or hyperspectral imaging as well as global navigation satellite systems are widely used for such purposes either aerially or at ground level (Rodriguez-Gonzálvez et al., 2017; Sánchez-Aparicio et al., 2018; Jo et al., 2019).

Due to the great variability of heritage environments in terms of dimensions, geometry, complexity and characteristics (Rodriguez-Gonzálvez et al., 2017), the hybridization of different acquisition techniques as well as the standardization of data collection and processing protocols would result in an improved digitalization of that places that guarantees a complete and good reconstruction. In this sense, not only the geometry and morphology of the historical assets should be analyzed, but also the physical and chemical characteristics in order to know their state of degradation. The great challenge is the integration of multi-source data acquired over the same area at different moments in order to monitor changes over time (Ioannides et al., 2018; Rodríguez-Gonzálvez et al., 2019).

This paper describes interdisciplinary research works performed in the Cueva Pintada in Galdar, Gran Canaria (Spain) where different geotechnologies were used in order to analyze their suitability and potential in the documentation of $\mathrm{CH}$ over time. Here, the importance of the 3D digitalization and reconstruction by means of sensor fusion is highlighted for the entire process of digital preservation of $\mathrm{CH}$ that is: data acquisition and processing as well as the representation, visualization and dissemination of final products.

\subsection{Study Case: Cueva Pintada}

The archaeological remains of the Cueva Pintada belong to a VIIXVI th centuries Canarian-Amazigh village (pre-Hispanic and contemporary of the first european settlement) located in Galdar, Gran Canaria (Spain) which was converted into a museum in 2006 (Onrubia-Pintado et al., 2016). Among the archaeological remains, the polychrome and decorated rock chamber attracts the most attention and gives its name to the museum (OnrubiaPintado et al., 2007). In this place, different research and innovation tasks are being developed with the aim of monitoring the degradation processes of the ruins as well as to aid in the decision making regarding the preservation and conservation of the heritage assets. This publication describes the principal documentation works performed in the archaeological remains by means of different sensors and techniques to document the historical site. The aim is to analyze the level of degradation of the different elements as well as to obtain a cartographic base for

\footnotetext{
* Corresponding author
} 
further studies. The products obtained serve to not only promote this heritage place by digital exhibition techniques but to perform further studies and aid in decision making thanks to a digital representation and management tool through which inspections of the artifacts can be performed.

With the goal of outline the 3D documentation and dissemination works performed in the Cueva Pintada, this paper is organized in four parts: After the introduction, section 2 focuses on the description of the technologies and techniques used for the data acquisition and processing, section 3 presents the products obtained regarding the 3D documentation, reconstruction and virtual simulation performed and finally, section 4 summarizes the main conclusions obtained after developing this works.

\section{MATERIALS AND METHODS}

\subsection{Material}

In order to accurately document the archaeological site and due to the level of detail required by the dual objective of obtaining a quality scientific and informative cartographic product, the following active and passive technologies were chosen for the data acquisition.

2.1.1 Terrestrial Laser Scanner: Regarding the active sensor, a Faro-Focus3D 120 (Figure 1a) which measures distances using the principle of phase shift at a wavelength of 905 $\mathrm{nm}$ was used. This device measures distances in the range of $0.60-120 \mathrm{~m}$ with a point measurement rate of 976.000 points per second. It has an accuracy of $0.015^{\circ}$ in normal lighting and reflectivity conditions and a beam divergence of $19 \mathrm{~mm}$ per $100 \mathrm{~m}$ range. The field of view extends $300^{\circ}$ vertically and $360^{\circ}$ horizontally with a $0.009^{\circ}$ of angular resolution, and the returning intensity is recorded in 11-bit format. Additionally, this laser scanner includes a double compensator in the horizontal and vertical axes.

As Figure 1a shows, a special piece (the yellow one) expressly designed for this work was arranged in the tripod so that the point of view of the TLS models coincided with that of the fisheye camera used for the panoramas generation (Figure 1b and Figure 3 ) in order to obtain a virtual tour with metric capacity. a)

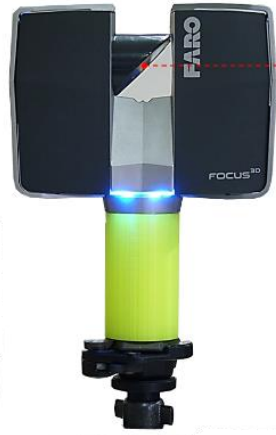

b)

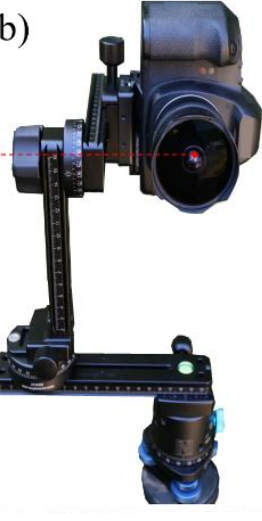

Figure 1. a) Faro-Focus3D 120 with the special piece designed to merge laser scanning models with panoramas and b) Fisheye camera.

\subsubsection{Pole Platform for Photogrammetric Acquisition:} Regarding the passive sensor, a Canon EOS 5D (Canon, Tokyo, Japan) was used mounted in a pole platform (Figure 2). This high-resolution SLR digital camera acquires RGB information with a resolution of $5616 \times 3744$ pixels. This camera uses a complementary metal-oxide-semiconductor sensor. The focal length is $24 \mathrm{~mm}$, and it shoots 3.9 frames per second.

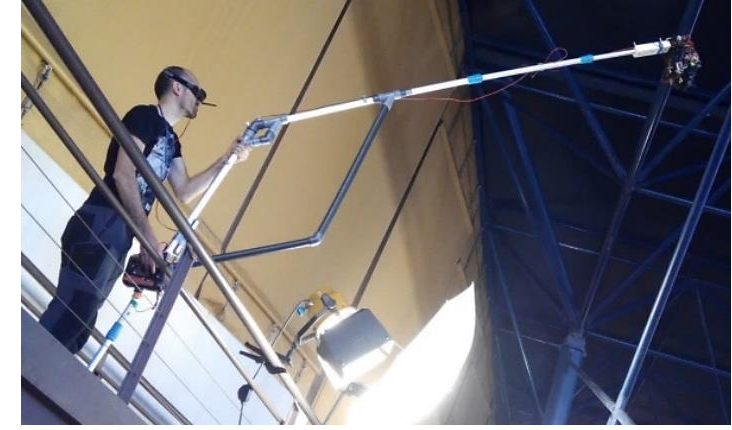

Figure 2. Pole platform employed for the photogrammetric data acquisition in Cueva Pintada.

In addition, a patented image acquisition system designed to acquire images with nadiral and oblique perspective (Figure 9) from a high point with respect to the ground was used. This pole platform is composed of an extendable structure in whose upper end a turntable stabilizing platform is placed where the image acquisition sensor is located. The lower end serves to support the system on the floor and to position the viewpoint of the camera in the desired location thanks to the extendable ergonomic frame available. To control the point of view of the camera the equipment is completed with a system of visualization of glasses and a remote control of the stabilizing turn platform.

2.1.3 Fisheye camera: The data acquisition for the generation of panoramas was performed by a Nikon D80 with a $180^{\circ}$ fisheye objective fixed to a tripod by a head that prevents parallax between consecutive images (Figure 3 ).

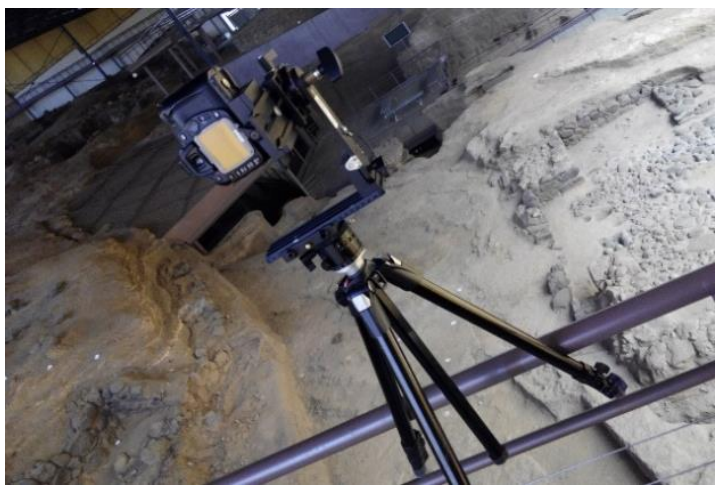

Figure 3. Image data acquisition with Nikon D80 for the generation of the virtual tour.

\subsection{Data acquisition}

2.2.1 Planning and Reference Network Design: In order to avoid occlusions and unnecessary data captured with both geotechnologies, a proper design of the scan and photogrammetric stations must be performed before the data collection. According to this design, an optimal number of data acquisition points are determined in such a way that there is no lack or excess of information. In addition, it is advisable to ensure a uniform lighting avoiding shadows to succeed in the photogrammetric processing and to ensure a homogeneous result in the virtual tour. Since the matter here is to obtain highresolution, accurate and photorealistic products by means of a correct hybridization of the multi data acquired from different platforms, a correct design of a reference network is the key to link data from both technologies. As Figure 4 shows, a reference network consisting of 16 spheres and 30 binary photogrammetric targets was designed to perform the data fusion. 


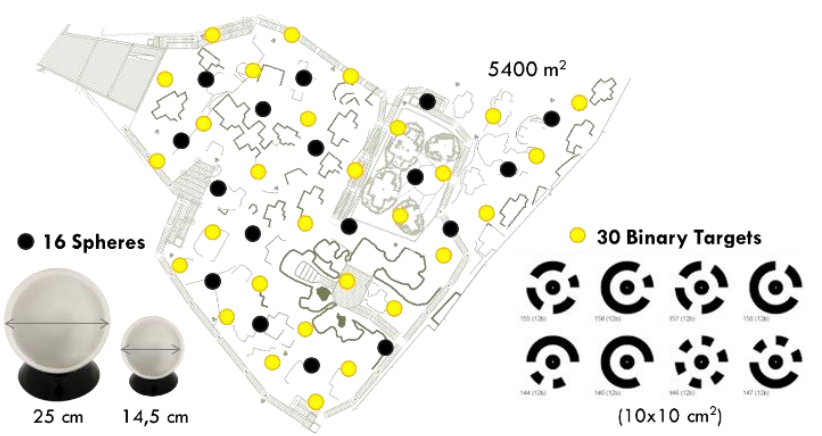

Figure 4. Reference network based on spheres and binary photogrammetric targets.

2.2.2 Laser Scanning Data Acquisition: Based on the number of laser stations established in the planning and the distance between them, a proper mesh step must be set in order to ensure the final resolution required for the laser scanning survey. The laser scanning data acquisitions were performed by mounting the Faro-Focus 3D 120 to a tripod and using a special piece designed to raise the TLS and place it at the same height and point of view of the camera used for the panorama data acquisition.

2.2.3 Photogrammetric Data Acquisition: concerning the high-resolution photogrammetric data acquisition and in order to ensure quality products, a proper protocol that considers some geometrical and radiometric restrictions is required. In this sense, a combination of a parallel and convergent protocol was applied (González-Aguilera et al., 2016) depending on the dimensions, geometry and complexity of each archaeological asset photographed. The overlap between consecutive image captures should be enough to the feature and the matching processing. In this case, an overlap of $80 \%$ between consecutive images was established.

2.2.4 Image Collection for the Panoramas' Generation: Uniform lighting conditions as well as proper ISO and aperture values are key to avoid noise and ensure an adequate depth of field. In addition, the number images required to cover the 360 horizontal and vertical degrees is dependent of the instrument employed. On the basis of the field of view offered by the fisheye objective used and the percentage of overlap chosen between consecutive images, the number of captures per station is calculated. In this case, even that the field of view offered by the fisheye lens used was $180^{\circ}$, seven images were taken to shape each panorama (six horizontal and one vertical) in order to ensure enough overlap. These was performed thanks to a tripod with a special head (Figure $1 \mathrm{~b}$ and Figure 3 ) through which the parallax between the seven images was avoided. After this process the panoramic image is generated (Figure 5).

\subsection{Pre-Processing}

2.3.1 Pre-Processing of TLS Point Clouds: The alignment between the different TLS stations was here performed thanks to the set of spheres and binary targets homogeneously distributed in the remains.

2.3.2 Image Pre-Processing: This step consists on obtaining the feature extraction and matching results between the set of images is obtained. The binary targets played here a key role by means of the key points detection and description as well as the image matching correspondence. Agisoft software was used to perform this process.

2.3.3 Panoramas Generation: Here, the relationship between the set of 7 images acquired at each station with different orientations is solved in order to obtain the final spherical image per station. To that end, a collinearity condition between Cartesian Coordinates and Polar Coordinates is established per pixel.

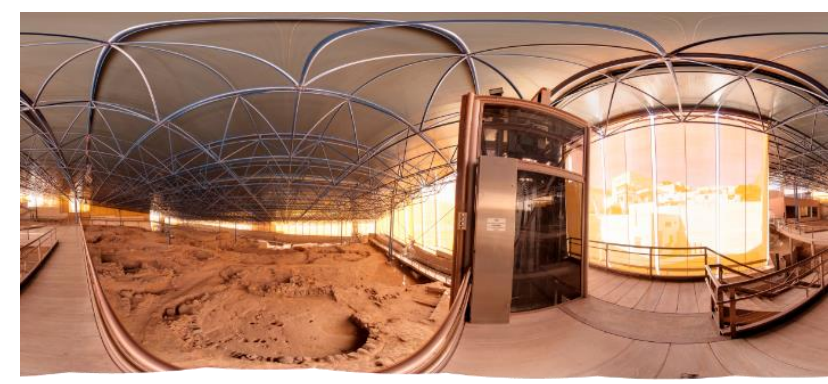

Figure 5. Example of a panorama obtained

\subsection{Processing:}

2.4.1 Processing of Laser Data: Regarding the processing of the laser data, several steps were carried out: (1) a distance filter was applied to homogenize the density of points of the final TLS model, (2) a voxelization (Figure 6) was applied to divide the TLS model in quadrants of $10 \times 10 \mathrm{~m}$ and help the next steps, (3) unnecessary points were manually removed per each quadrant and finally, (4) a decimation of the model was performed at two resolutions to meet the established requirements for the final products.

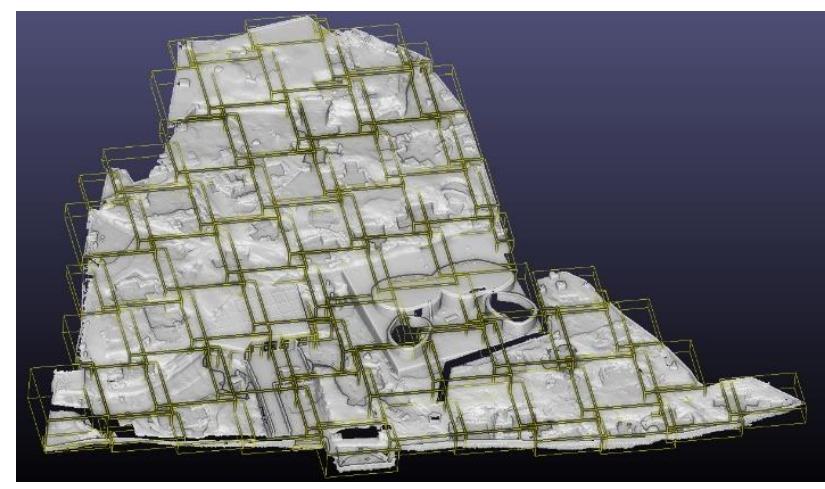

Figure 6. Voxelization applied to the laser scanning 3D model.

2.4.2 Photogrammetric 3D Model Generation: This process goes through several steps: (1) the relative orientation and self-calibration of the camera at each point, (2) the absolute orientation of each camera location and (3) the dense matching where the 3D point cloud model is obtained. 


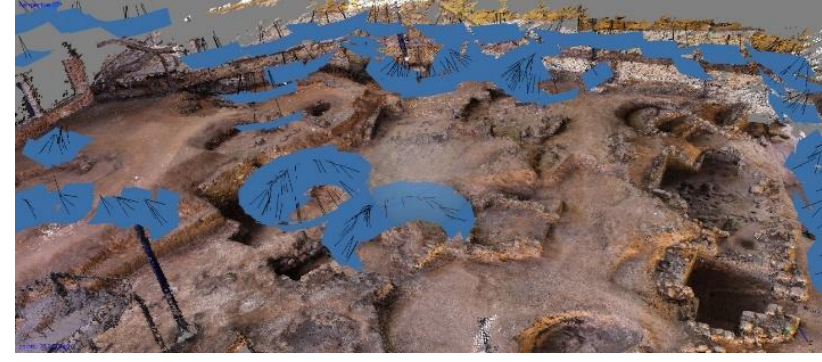

Figure 7. Photogrammetric model in which each camera position is displayed in blue.

2.4.3 Virtual Tour Generation: This is the final visual interface for studying the archaeological heritage site. This tool was developed using a web-based spherical virtual tour based on Java technology. The stations were arranged so that there were no hidden areas and considering that the areas of greatest interest of the archaeological site are displayed. Here, hybrid products derived from the laser scanning and the photogrammetric processing such as orthoimages, 3D models and images of detailed can be incorporated. All information is loaded in the same virtual environment with a map of the archaeological site in such a way that the user is oriented with respect each virtual position regarding the entire archaeological site.

\section{RESULTS}

To document the approximately $5400 \mathrm{~m}^{2}$ of the Cueva Pintada archaeological site, two surveys were carried out in order to generate the photorealistic 3D model of the site: (i) a laser scanner survey and (ii) a photogrammetric survey. Finally, an acquisition of panoramas was carried out in order to merge all the data and have a virtual environment for both scientific and educational purposes.

\subsection{Laser Scanner Survey}

229 TLS scans were performed with an approximate resolution of $6 \mathrm{~mm}$ at 10 meters distance, equivalent to 44 million points per station. The nominal precision was $\pm 1.5 \mathrm{~mm}$. A total of 10100 million points were captured with the Faro-Focus3D 120 (Figure 8).

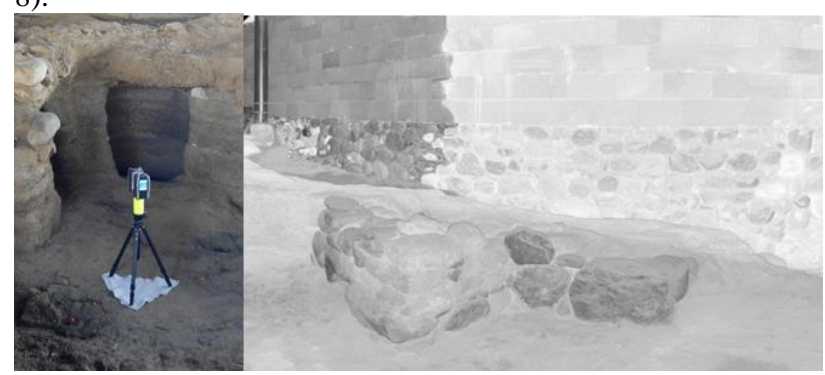

Figure 8. Data acquisition with the Faro-Focus3D 120 (Left) and the corresponding range image obtained (Right).

\subsection{Photogrammetric Survey}

For the photogrammetric survey, a specific acquisition system was expressly designed (Figure 2) where a Canon EOS 5D reflex camera of 21 megapixels was embarked on a self-stabilized platform. Regarding the configuration parameters, a fixed focal length of $20 \mathrm{~mm}$, and a height between 2 and 4 meters was established. This is the reason why the GSD of images went from 0.6 to $1.2 \mathrm{~mm}$. Given the heterogeneity of the archaeological site, as well as some access limitations due to conservation conditions, it was not feasible to use a standardized aerial photogrammetric acquisition protocol. Therefore, both the height and the angle of each image acquisition were chosen according to the characteristics of each individual area, lighting conditions, complexity of the structures and the access restrictions. 3321 photographs were taken in RAW format, of which 2728 were preselected.

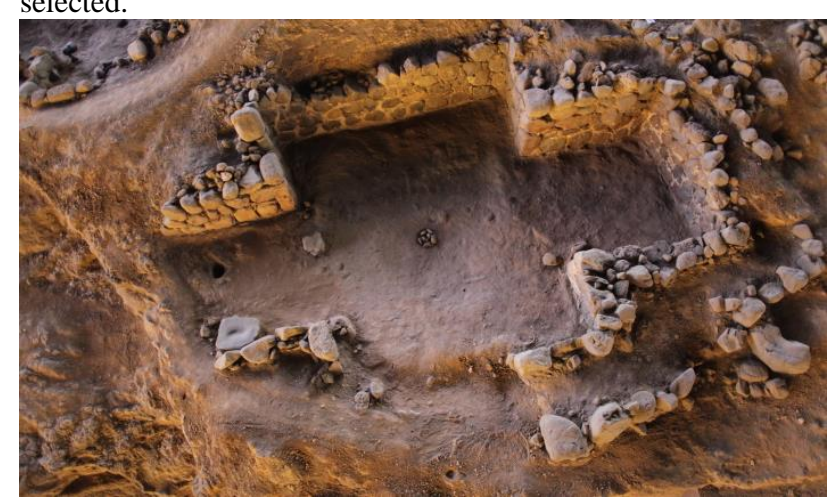

Figure 9. Example of a nadiral and oblique image acquired from the pole platform system.

\subsection{Virtual Tour with Metric Properties}

In relation to the photogrammetric survey for the virtual visit generation, 103 photogrammetric stations were established for the generation of $360^{\circ}$ immersive images. This redundancy responds to the variations of light conditions within the reservoir, which allowed selecting the optimal ones for the virtual visit. Given particular light conditions, image acquisitions were made in RAW format (*.NEF), with manual bracketing, according to the conditions of each area and taking into account the instantaneous lighting. Specifically, 2770 images were taken for the 103 station points, composed of 7 images and an average of 3.84 photographs per station point. 28 of the 103 images corresponded to the same laser scanner station points so that linking the laser model to the panoramic images was possible.

\subsection{Multi-Data Source Fusion}

Based on the reference network established during the fieldwork, all laser scans were aligned in the same local reference system in order to guarantee the highest possible accuracy. For this work, a total of 67 reference spheres were identified in the 229 scans performed, with an error of less than $2 \mathrm{~mm}$. Note that although 16 reference spheres were available in the field (Figure 4), by using specially designed anchoring systems, it was possible to move them according to the needs of each area of the reservoir, thus increasing their total effective number. In order to verify the alignment errors, the coordinates of the topographic system previously established at some points in the archaeological site were contrasted with those obtained from such points with the laser model. The results are shown in Table 1. A posteriori deviation of \pm 0.001689 m was obtained with approximately \pm $1.7 \mathrm{~mm}$ accuracy, verifying that the error of the local laser system meets the specifications of the job (error less than $2 \mathrm{~mm}$ ).

\begin{tabular}{llll}
\hline Parameters & & Value & Accuracy \\
\hline \multirow{2}{*}{ Translation } & X-Coordinate & 435679.943256 & \pm 0.001063 \\
$(\mathrm{~m})$ & Y-Coordinate & 3113299.081237 & \pm 0.000741 \\
& Z-Coordinate & 112.164689 & \pm 0.001560 \\
\hline \multirow{2}{*}{ Rotation } & X-Axis $(\omega)$ & -0.00000176 & \pm 0.00004035 \\
$(\mathrm{rad})$ & Y-Axis $(\varphi)$ & +0.00017037 & \pm 0.00005154 \\
& Z-Axis $(\kappa)$ & +0.42133813 & \pm 0.00002850 \\
\hline Scale & & \multicolumn{1}{c}{1} & - \\
\hline
\end{tabular}

Table 1. Estimated values and associated precision of the parameters of the transformation between both reference systems. 
Once the scans were aligned and the coordinates of each laser point station was established, the individual debugging of each scan was carried out, establishing two different phases. First, all those points whose distance to the centre of projection was greater than 8 meters for those areas where there was a presence of archaeological remains (maximum spatial resolution of 4.9 $\mathrm{mm}$ ) and of 16 meters in the rest (maximum resolution) were filtered by a distance of $9.8 \mathrm{~mm}$ ). Next, those points unnecessary for the documentation of the archaeological remains, such as gateways, reference elements and other artificial structures, were eliminated. The result was of approximately 3,600 million points (Figure 10).

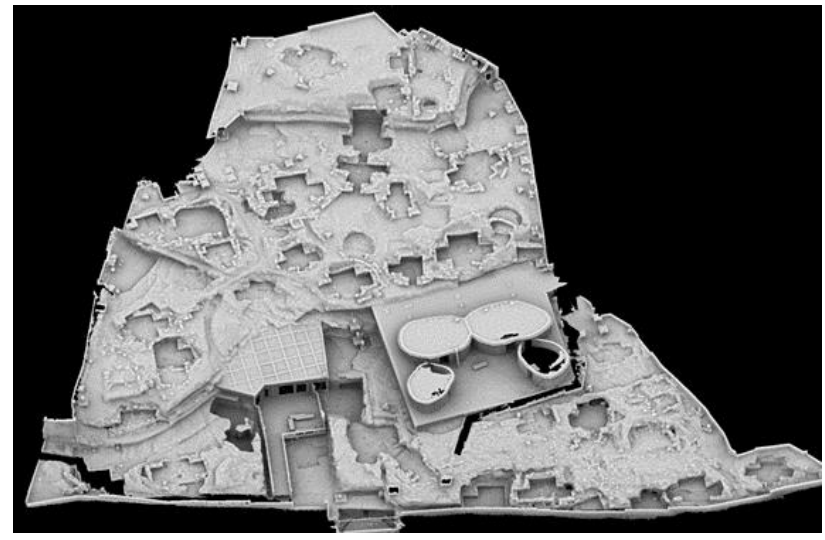

Figure 10. General view of the laser model.

For the processing of the data corresponding to the aerial photogrammetric survey, a preselection of the original images was made taking into account the quality of the focus, the exposure and the shooting angles. To facilitate processing, the selected images were separated into three groups. These groups have the necessary overlap for their subsequent fusion in order to generate the final geomatic product. Once the images of the three groups were aligned, the densification of the point clouds, the generation of the triangle meshes and the assignment of textures to them were performed.

On the previous texturized model, the targets used for georeferencing (coordinates from laser scans) were identified and the projection of each of these targets were refined in the images in which they appear. Among all the targets used, a selection of the most favourable ones was made in terms of spatial distribution and the number of images in which they appeared. As a result of the selection, 10 targets were used. The mean error of the georeferencing process was $9.901 \mathrm{~mm}$. After georeferencing, the generation of the dense point cloud and the mesh of definitive triangles was proceeded. Then the three triangle meshes from the three groups of images were merged into one and, in order to achieve greater homogeneity, the textures for this resulting mesh were generated. The following table (Table 2) summarizes the results of the point cloud and the corresponding mesh.

\begin{tabular}{ll}
\hline Parameters & Value \\
\hline No. of points & $71,768,099$ \\
No. of mesh triangles & $3,687,843$ \\
Size of the texturized model (pixels) & $16,384 \times 16,384$ \\
GSD (mm/pixel) & 1.47 \\
\hline
\end{tabular}

Table 2. Summary of the products derived from the photogrammetric model.
The laser scanning model of the remains was merged with the photogrammetric data in order to generate a final homogeneous product (Figure 11). This last phase involves mainly a radiometric correction of the RGB values captured in the field by the TLS.

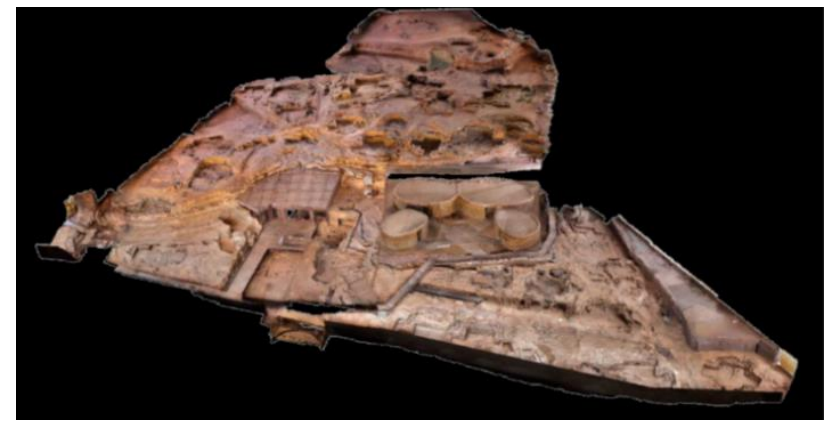

Figure 11. 3D model of the Cave Painted archaeological park with photorealistic quality.

In relation to the virtual tour, the images acquired in the field will be revealed to convert them from RAW format to TIFF format. Subsequently they were semi-automatically aligned with each other, through a detection of points of interest and subsequent manual filtering, for avoiding errors. These panoramas were the framework of the virtual visit.

The virtual tour consisted of 69 panorama stations, linked by 859 links (Figure 12). It also included external links for additional resources, such as links to other linked information, prepositioned in the areas of audio-visual resources in the site. These resources can be composed of text, videos and/or images as required by the museum management. The result of the visit included, along with the informative, a scientific component based on the possibility of making geometric measurements on the panoramas (Figure 13).

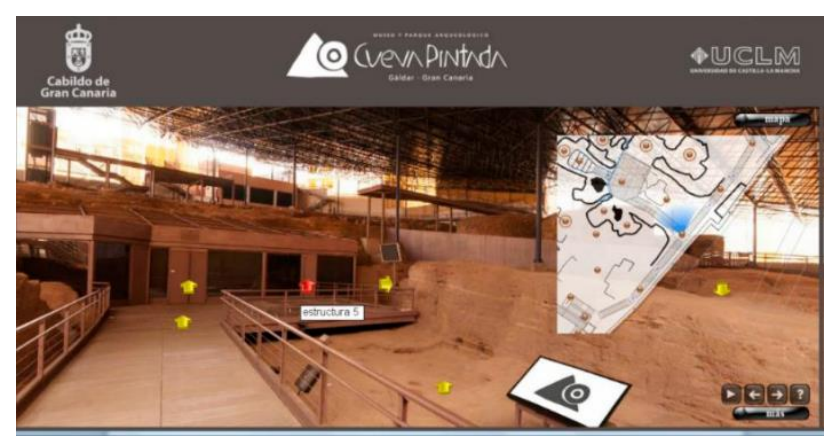

Figure 12. Virtual end visit of the Cave Painted archaeological site (right).

The final virtual visit not only incorporated conventional panoramas, oriented to divulgation purposes, but also a series of selected metric panoramas in certain areas of the site (Figure 13). 28 of the 229 TLS stations were made in such a way that their point of view of the camera matched to achieve metric panoramas. Given the very nature of this derived product, as well as its scientific purpose, its visualization was not direct in the virtual visit itself, being necessary a third-party software. 


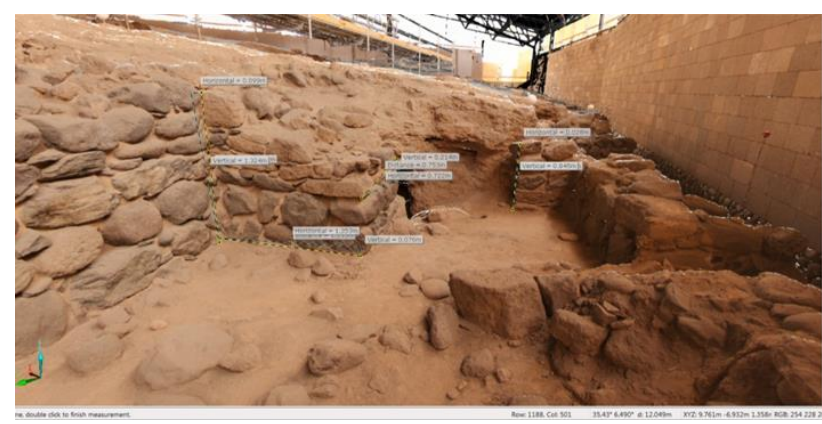

Figure 13. Example of the metric panorama corresponding to structure 12 .

\section{CONCLUSIONS}

The combination of laser scanning and close-range photogrammetry provide valuable solutions when monitoring, conservation, restoration and protection of archaeological sites are needed. They allow faithfully documenting the remains and obtaining a 3D reconstruction of the place very suitable for virtual visualization and dissemination. However, the delicate conditions to perform the data collection might be highlighted. The advantage of the proposed methodology is the accuracy that can be achieved in a relatively short time. Even though closerange scanners have better accuracy, they increase the fieldwork considerably (to a nearly unachievable level) if the entire archaeological site must be documented. With this type of reconstruction, it is possible to obtain both an accurate metric and the ability for the user to explore scenarios in ways that are not allowed in reality, such as: access to areas that are restricted due to storage conditions, or access to areas that are totally closed to the public due to legal status and/or to preserve the original condition. This study highlights the advantages that the combination of both techniques has, in terms of accuracy, time, production and 3D results, as opposed to traditional techniques. Diffusion through the virtual tour provides thousands of users' access to the archaeological site despite the physical access restrictions.

\section{ACKNOWLEDGEMENTS}

Authors would like to thank Cabildo of Gran Canaria and University of Castilla-La Mancha allowing us to access to the archaeological site of Cueva Pintada as well as to facilitate the work developed there. Specially thanks the University of Castilla-La Mancha for the support given and the facilities offered when lending their equipment.

\section{REFERENCES}

Giannini, M., Castagnetti, C., and Rivola, R., 2017. WebGIS, 3D modeling and virtual tours to map, record and visualize the cultural, archaeological and landscape heritage: the visualversilia project, 3D surveying. In: 3rd IMEKO International Conference on Metrology for Archaeology and Cultural Heritage. Vol. 3, pp. 166-171.

González-Aguilera, D., López-Fernández, L., RodriguezGonzalvez, P., Guerrero, D., Hernandez-Lopez, D., Remondino, F., and Gaiani, M., 2016. Development of an all-purpose free photogrammetric tool. In: The International Archives of the Photogrammetry, Remote Sensing and Spatial Information Sciences, Vol. XLI-B6, 4.
Hua, L., Chen, C., Fang, H., and Wang, X., 2018. 3D documentation on Chinese hakka tulou and internet-based virtual experience for cultural tourism: a case study of yongding county, Fujian. Journal of Cultural Heritage, 29, pp. 173-179.

Hoffmeister, D., 2017. Simulation of tallow lamp light within the $3 \mathrm{~d}$ model of the Ardales cave, Spain. Quaternary International, 430 , pp. 22-29.

Ioannides, M., Fink, E., Brumana, R., Patias, P., Doulamis, A., Martins, J., \& Wallace, M., 2018. Digital Heritage. Progress in Cultural Heritage: Documentation, Preservation, and Protection: 7th International Conference, EuroMed 2018, Nicosia, Cyprus, October 29-November 3, 2018, Proceedings Springer. Vol. 11196.

Jo, Y. H., and Hong, S., 2019. Three-dimensional digital documentation of cultural heritage site based on the convergence of terrestrial laser scanning and unmanned aerial vehicle photogrammetry. ISPRS International Journal of GeoInformation, 8(2), 53. doi.org/10.3390/ijgi8020053

Khodeir, L. M., Aly, D., and Tarek, S., 2016. Integrating HBIM (heritage building information modelling) tools in the application of sustainable retrofitting of heritage buildings in Egypt. Procedia Environmental Sciences, 34, pp.258-270. doi.org/10.1016/j.proenv.2016.04.024

Landeschi, G., Apel, J., Lundström, V., Storå, J., Lindgren, S., and Dell'Unto, N., 2018. Re-Enacting the Sequence: Combined Digital Methods to Study a Prehistoric Cave. Archaeological and Anthropological Sciences, pp. 1-15.

Lerma, J. L., Navarro, S., Cabrelles, M., and Villaverde, V., 2010. Terrestrial laser scanning and close-range photogrammetry for 3D archaeological documentation: the Upper Palaeolithic Cave of Parpalló as a case study. Journal of Archaeological Science, 37(3), pp. 499-507.

Lucci, E., di Lernia, S., Monaco, A., Jnen, M., and Nasr, J. B., 2019. Prehistoric and Historic Monumental Funerary Structures in the "Chott El Jérid" Area (Southern Tunisia): The Importance of Photogrammetry for Rapid and Complete Documentation in Saharan Contexts.

Megahed, N., 2015. Towards a theoretical framework for HBIM approach in historic preservation and management. International Journal of Architectural Research: ArchNet-IJAR, 9(3), pp. 130147.

Niccolucci, F., 2016. Setting standards for 3d visualization of cultural heritage in Europe and beyond. In: Paradata and Transparency in Virtual Heritage. Routledge, pp. 49-62.

Onrubia-Pintado, J., Rodríguez-Santana, C. G., Sáenz-Sagasti, J. I., and Antonia del Val, V., 2007. El museo y parque arqueológico cueva pintada (Gáldar, Gran Canaria): de manzana agrícola a parque arqueológico urbano. In: IV Congreso Internacional sobre Musealización de Xacementos Arqueolóxicos: Conservación e Presentación de Xacementos Arqueolóxicos no Medio Rural. Impacto Social no Territorio. Dirección Xeral De Patrimonio Cultural, pp. 183-190.

Onrubia-Pintado, J., Sáenz-Sagasti, J. I., Rodríguez-Santana, C. G., Hernández-López, D., González-Piqueras, J., QuintanillaRódenas, A., Vicent-García, J.M., Espadas-Álvarez, C., González-Aguilera, D., Guerrero-Sevilla, D., Marchante-Ortega, 
Á. and Rodríguez-Gonzálvez, P., 2016. Nuevas técnicas para la investigación y la conservación de la cueva pintada de Gáldar (Gran Canaria); modelado 3D y análisis de imagen. In: XXII Coloquio de Historia Canario-Americana. Las Palmas de Gran Canaria: Ediciones del Cabildo De Gran Canaria. XXII-183, pp.1-20.

Patias, P., Sylaiou, S., Sechidis, L., Spartalis, I., Grussenmeyer, P., Meyer, E., Landes, T., Alby, E., 2006. A proposed low-cost system for 3D archaeological documentation. In: Proceedings of the $7^{\text {th }}$ International Symposium on Virtual Reality, Archaeology and Cultural Heritage, Nicosia, Cyprus, pp. 145-149.

Pozzi-Escot, D., Oshiro, J., Romano, G., Capozzoli, L., Lasaponara, R., and Masini, N., 2018. Traces in the desert: use of new technologies for the study and valorization of the Pachacamac sanctuary - Lima, Peru. Heritage Science, 6(1), 68.

Rodríguez-Gonzálvez, P., Muñoz-Nieto, A. L., del Pozo, S., Sanchez-Aparicio, L. J., González-Aguilera, D., Micoli, L.and Haynes, I., 2017. 4D reconstruction and visualization of cultural heritage: analyzing our legacy through time. In: The International Archives of Photogrammetry, Remote Sensing and Spatial Information Sciences, 42, 609.

Rodríguez-Gonzálvez, P., Guerra Campo, Á., Muñoz-Nieto, Á. L., Sánchez-Aparicio, L. J., and González-Aguilera, D., 2019. Diachronic reconstruction and visualization of lost cultural heritage sites. ISPRS International Journal of Geo-Information, $8(2), 61$.

Rubinowicz, P., and Czyńska, K., 2015. Study of city landscape heritage using lidar data and 3d-city models. In: International Archives of the Photogrammetry, Remote Sensing and Spatial Information Sciences. Vol. XL-7/W3.

Sánchez-Aparicio, L. J., Del Pozo, S., Ramos, L. F., Arce, A., and Fernandes, F. M., 2018. Heritage site preservation with combined radiometric and geometric analysis of TLS data. Automation in Construction, 85, pp.24-39.

Themistocleous, K., 2017. Model reconstruction for 3D vizualization of cultural heritage sites using open data from social media: the case study of Soli, Cyprus. Journal of Archaeological Science: Reports, 14, pp.774-781. 